\title{
Accounting for immediate emotional memory enhancement
}

\author{
Deborah Talmi ${ }^{\mathrm{a}, \mathrm{b}, *}$, Lucy M. McGarry ${ }^{\mathrm{b}}$ \\ a School of Psychological Sciences, University of Manchester, UK \\ ${ }^{\mathrm{b}}$ Department of Psychology, University of Toronto, Canada
}

\section{A R T I C L E I N F O}

\section{Article history:}

Received 2 November 2010

revision received 24 July 2011

Available online $\mathrm{xxxx}$

\section{Keywords:}

Emotion

Free recall memory

Divided attention

Distinctiveness

Semantic relatedness

\begin{abstract}
A B S T R A C T
Memory for emotional events is usually very good even when tested shortly after study, before it is altered by the influence of emotional arousal on consolidation. Immediate emotion-enhanced memory may stem from the influence of emotion on cognitive processes at encoding and retrieval. Our goal was to test which cognitive factors are necessary and sufficient to account for EEM, with a specific focus on clarifying the contribution of attention to this effect. In two experiments, participants encoded negative-arousing and neutral pictures. In Experiment 1, under divided-attention conditions, negative pictures were better attended and recalled even when they were matched with neutral pictures on semantic relatedness and distinctiveness, and attention at encoding predicted subsequent emotion-enhanced memory. The memory advantage for emotional stimuli was only abolished when attention to emotional and neutral stimuli was also matched, under full-attention in Experiment 1 and under divided-attention in Experiment 2. Emotional memory enhancement was larger in Experiment 1 when the control of organization and distinctiveness was relaxed. These findings suggest that attention, organization and distinctiveness provide a necessary and sufficient account for immediate emotion-enhanced free recall memory.
\end{abstract}

(c) 2011 Elsevier Inc. All rights reserved.

\section{Introduction}

Memory for moderately arousing emotional stimuli, such as images of violence, is better than memory for neutral stimuli. There is good evidence for emotion-enhanced memory (EEM) in both humans and non-human animals (Cahill \& McGaugh, 1998; Labar \& Cabeza, 2006). Evidence from animals shows that the sympathetic emotional arousal response enhances long-term memory by activating the amygdala, which modulates the long-term consolidation of memory traces in the hippocampus, so that after a prolonged delay, memory for emotional events is enhanced (McGaugh, 2004). Although this model can explain a host of data from human participants (Labar \& Cabeza, 2006), researchers often overlook the fact that because the modulation mechanism only influences long-term memory

\footnotetext{
* Corresponding author at: School of Psychological Sciences, University of Manchester, Manchester M13 9PL, UK. Fax: +44 (0) 1612752623.

E-mail address: Deborah.Talmi@manchester.ac.uk (D. Talmi).
}

consolidation, it does not account for the enhanced memory in immediate long-term memory tests (Cahill \& McGaugh, 1998), namely tests that occur shortly after study but following a brief distractor activity, which clears working memory. In the general discussion section we review evidence from animal studies that shows conclusively that the mechanism used by the modulation model to account for delayed EEM does not account for immediate EEM (Bianchin, Mello e Souza, Medina, \& Izquierdo, 1999; Ellis \& Kesner, 1983; Frey, Bergado-Rosado, Seidenbecher, Pape, \& Frey, 2001; Seidenbecher, Reymann, \& Balschun, 1997). A complementary mechanism is therefore required to account for immediate EEM, which humans exhibit readily.

The goal of the current study was to establish a cognitive account of immediate EEM. The cognitive account attributes this effect to altered encoding and retrieval of emotionally arousing events, instead of to their modulated consolidation. The notion that cognitive factors contribute to immediate EEM is not new (Cahill \& McGaugh, 1998; Kensinger \& Corkin, 2004), yet it is unknown which factors 
are necessary and sufficient to account for this effect. Consequently, the cognitive account has had a relatively modest influence on neuroscience research. For example, a recent meta-analysis (Murty, Ritchey, Adcock, \& Labar, 2010) highlighted the fact that although many brain regions are consistently associated with EEM, their contribution to EEM is under-investigated. This is likely due to the prominence of the modulation model, as researchers typically focus on brain regions relevant to that model and interpret their findings within its framework even when memory is tested shortly after study (Kensinger \& Corkin, 2004; Sommer, Glascher, Moritz, \& Buchel, 2008; Strange, Hurlemann, \& Dolan, 2003). Moreover, researchers interested in modulated consolidation test memory after a prolonged delay, which complicates the isolation of an independent cognitive contribution (Ritchey, BessetteSymons, Hayes, \& Cabeza, 2011). Understanding the critical psychological determinants of immediate EEM can inspire and inform future research of its underlying brain mechanisms.

Our objective here was to show that the influence of three factors - organization, distinctiveness, and attention - on encoding and retrieval provides a necessary and sufficient account of immediate EEM in free recall. The two experiments reported here support this claim by showing that EEM can only be abolished when these three factors are controlled. Our approach relies on the assumption that to fully understand an empirical phenomenon, such as immediate EEM, we need to know the conditions for its manifestation, and that such understanding is evident in the ability to systematically influence the phenomenon by manipulating its triggering conditions.

The contribution of organization, distinctiveness, and attention to EEM will be reviewed next.

\section{Organization}

Organization is operationalized here simply as the semantic cohesiveness or inter-relatedness of a stimulus set. Organization improves memory (Hunt \& McDaniel, 1993; Mandler, 1967) because it encourages inter-item elaboration at encoding (Einstein \& Hunt, 1980) and serves as a cue at retrieval (Tulving \& Pearlstone, 1966). Emotional events are often related to each other. They may belong to the same script, such as the sound of emergency sirens and the image of a smashed car; or be related thematically, as when thinking about poverty conjures up images of hungry children. This feature of emotional events is captured in the laboratory because experimental stimulus sets often include inter-related emotional stimuli, such as the words 'torture' and 'suicide', alongside unrelated neutral stimuli (Maratos, Allan, \& Rugg, 2000; Talmi \& Moscovitch, 2004).

When emotional, non-taboo words were compared to equally-related neutral words, participants recalled the neutral words as well as the emotional words (Talmi \& Moscovitch, 2004), but follow-up work showed that organization only partly accounts for EEM. When more arousing stimuli were used, such as taboo words or pictures (De Houwer \& Hermans, 1994; Kensinger \& Schacter, 2006), controlling organization attenuated EEM in free recall but did not abolished it completely (Buchanan, Etzel, Adolphs, \& Tranel, 2006; Talmi, Luk, McGarry, \& Moscovitch, 2007; Talmi, Schimmack, Paterson, \& Moscovitch, 2007). The semantic relatedness of emotional words also influences bias in recognition memory tests, where participants exhibit increased propensity to endorse emotional lures as 'old'; but its influence on recognition accuracy is less well established (Maratos et al., 2000; Windmann \& Kutas, 2001). The finding that the magnitude of EEM depends on the semantic relatedness of neutral stimuli suggests that organization contributes to EEM. However, because these studies did not manipulate the semantic relatedness of emotional stimuli, they do not conclusively demonstrate that organization contributes to memory for emotional information.

\section{Distinctiveness}

Distinctiveness is not an inherent property of a stimulus, but a feature of the context in which it is embedded. Schmidt (1991) argued that emotional stimuli are distinct relative to the content of participants' long-term memory because they have unique attributes that they do not share with most stored stimuli, which are neutral. This form of distinctiveness, relative to stimuli stored in long-term memory, is termed secondary distinctiveness (Hunt \& Worthen, 2006; Schmidt, 1991). Emotional stimuli also stand out relative to the neutral stimuli that typically surround them at the time of encoding, for example, neutral stimuli in the same study list or the peripheral details of a crime scene (Schmidt, 1991). Distinctiveness relative to stimuli stored in working memory is termed primary distinctiveness (Hunt \& Worthen, 2006; Schmidt, 1991). To clarify, a picture of a nude model within a set of pictures of clothed models has both primary and secondary distinctiveness, but a picture of a clothed model within a series of nudes only has primary distinctiveness (Schmidt, 2002). There is strong evidence that primary distinctiveness improves memory, but that secondary distinctiveness does not (Hunt \& McDaniel, 1993; Schmidt, 1991). For example, common sentences are remembered as well as sentences with high secondary distinctiveness, such as bizarre or humorous sentences, when each sentence type is presented to a separate group of participants or in separate blocks to the same participants. However, when all sentences are mixed and presented to the same group of participants, the bizarre or humorous sentences are remembered better than the neutral ones, an effect which must therefore stem from their primary distinctiveness (McDaniel, Einstein, Delosh, May, \& Brady, 1995; Schmidt, 1994).

Emotional stimuli always have high secondary distinctiveness, but their primary distinctiveness can be manipulated by varying the composition of experimental stimulus sets. The primary distinctiveness of emotional stimuli is enhanced relative to the primary distinctiveness of neutral stimuli when the same experimental list includes a small number of emotional stimuli intermixed with a larger number of neutral stimuli. By contrast, both stimulus types have equivalent primary distinctiveness when they are presented in 'blocked' sets that only contain other stimuli 
of the same type. A number of studies blocked stimulus type in this manner and found that EEM was abolished (Dewhurst \& Parry, 2000; Hadley \& Mackay, 2006; Sommer et al., 2008; Talmi \& Moscovitch, 2004). When organization was also controlled, the same results were obtained with more arousing pictures (Talmi, Luk, et al., 2007). The comparison between blocked and mixed set results suggests that primary distinctiveness plays a role in EEM. A contradictory result was obtained in two other studies (Majerus \& D’Argembeau, 2011; Monnier \& Syssau, 2008, Experiment 2), which found EEM for positive words even when stimulus type was blocked. Some methodological differences may account for this difference results. These two studies focused on short-term memory, and therefore employed short lists and a serial recall test. Monnier and Syssau did not control semantic relatedness, and although the semantic relatedness of all word types was very low in the Majerus and D'Argembeau experiment, the positive words were more inter-related than the neutral ones. Notably, Majerus and D'Argembeau did not obtain EEM for negative words.

The finding that EEM can be attenuated by blocking stimulus type at encoding is important because it allows us to discard two alternative accounts of immediate EEM. The first is the suggestion that immediate EEM depends on arousal, just like delayed EEM. Had that been the case EEM should have also been obtained under blocked-sets conditions, because physiological measures of emotional arousal are sustained and even increase after exposure to a series of unpleasant pictures (Smith, Bradley, \& Lang, 2005).

Second, the results of the Talmi, Luk, et al. (2007) study also contradicted an explicit prediction of the Binding Theory of EEM (Hadley \& Mackay, 2006; Mackay et al., 2004). According to this theory the amygdala's response to emotional arousal allows emotional stimuli to gain privileged access to 'binding nodes'. Binding nodes link items to their episodic context and facilitate their subsequent retrieval. When items follow each other quickly the binding of emotional stimuli is thought to be prioritized so that neutral stimuli that immediately follow or precede an emotional stimulus would be bound less well to the context, and consequently remembered less well. Binding Theory thus appears to account for the finding that EEM is obtained under mixed-set but abolished under blocked-set conditions (Hadley \& Mackay, 2006). Crucially, however, when a slow presentation rate is used (above $2000 \mathrm{~ms}$ per item) Binding Theory explicitly predicts that memory for neutral items would improve in mixed-set relative to blocked-set conditions (Hadley \& Mackay, 2006, p. 84), because a slow presentation rate gives all items sufficient time to be bound, and the presence of emotional items would further strengthen the sequential links between neutral items and their near neighbors to facilitate retrieval. This prediction was falsified by Talmi, Luk, et al. (2007), who used a slow presentation rate and found poorer memory for neutral stimuli under mixed-set relative to blocked-set conditions. In her review, Mather (2007) concludes that Binding Theory does not currently account for the consistency with which primary distinctiveness results in EEM across fast and slow presentation rates.
How primary distinctiveness influences memory is under debate (Hunt \& McDaniel, 1993), but experimental evidence suggests that it does so at retrieval (Hunt \& Worthen, 2006), when distinct items, which have more unique attributes, are more likely to be recovered following memory search (Tomlinson, Huber, Rieth, \& Davelaar, 2009). A retrieval effect is in line with the stronger influence of primary distinctiveness on free recall relative to recognition memory tests (Schmidt, 1991) and with the finding that primary distinctiveness enhances memory even when it is only manipulated at retrieval. For example, when participants encode two sets of stimuli separately, one higher and one lower in secondary distinctiveness, they recall more of the stimuli that are higher in secondary distinctiveness when they recall both sets together - a situation in which these stimuli are also higher in primary distinctiveness - than when they recall them separately (Talmi, Luk, et al., 2007; McDaniel, Dornburg, \& Guynn, 2005). A retrieval effect is also in line with the finding that manipulating set composition does not alter memory for emotional stimuli, (Hadley \& Mackay, 2006; Talmi, Luk, et al., 2007), as would be expected if primary distinctiveness operated at encoding. To gain insight into the stage with which the primary distinctiveness of emotional stimuli influences EEM Experiment 1 will relate the amount of attention captured at encoding and subsequently enhanced memory under both mixed- and blocked-set composition conditions.

\section{Attention}

The capacity theory of attention (Kahneman, 1973) assumes that people's ability to perform mental work is limited, but that they can normally allocate their limited capacity freely among concurrent activities (Craik, Govoni, Naveh-Benjamin, \& Anderson, 1996). According to this theory, impaired performance on a secondary task in a divided attention paradigm is a measure of the attention resources allocated to the primary task (Craik et al., 1996; Eysenck \& Eysenck, 1979; Kahneman, 1973; Kerr, 1973). When attention resources are insufficient to meet task demands enhanced attention at encoding increases the likelihood that encoding-promoting cognitive processes would take place (Mitchell \& Hunt, 1989).

Emotional stimuli capture more attention than neutral stimuli (Vuilleumier, 2005) despite participants' explicit goals. This is evident in longer latency and reduced accuracy in prioritized concurrent tasks when participants encode emotional stimuli, relative to when they encode neutral stimuli (Schimmack, 2005; Talmi, Schimmack, et al., 2007). It is also evident in longer latency to respond to emotional stimuli in a variety of paradigms where a neutral goal competes with the processing of emotional stimuli, such as reading, lexical decision, dot-probe and Stroop tasks (Algom, Chajut, \& Lev, 2004; Anderson, 2005; Hadley \& Mackay, 2006; Mackay et al., 2004; Mogg, Bradley, de Bono, \& Painter, 1997; Schmidt \& Saari, 2007; Talmi, Schimmack, et al., 2007). In those tasks emotional stimuli are preferentially attended despite other task demands.

Arguably, the effect of emotion on attention could either occur in parallel to its effect on memory, or it 
could mediate the memory effect. The research we will review below will show that surprisingly, there is no conclusive evidence to decide between these two possibilities.

In order to decide between them, we could utilize situations in which capacity is nearly exhausted, because in those situations attention to emotional and neutral stimuli may be almost equivalent. This occurred, for instance, in two studies that employed an incidental encoding paradigm and presented emotional and neutral words very briefly (Hadley \& Mackay, 2006; Sharot \& Phelps, 2004). If attention mediates EEM then EEM in these situations should be abolished, and indeed, both studies did not find EEM in an immediate memory test. However, because neither study measured attention, this interpretation is inconclusive.

Another way of testing which of the two possibilities is correct is to utilize situations in which it is likely that emotional stimuli would receive more attention than neutral stimuli. This occurs, for instance, under divided-attention conditions, and indeed, a number of studies found that EEM is larger under divided- attention relative to fullattention conditions (Kensinger \& Corkin, 2004; Kern, Libkuman, Otani, \& Holmes, 2005). Another study (Majerus \& D'Argembeau, 2011) manipulated the distinctiveness of emotional stimuli by changing their frequency in short mixed sets. The authors hypothesized that when emotional stimuli are infrequent they receive more attention at encoding and, when no distractors are interpolated between study and test, they can be maintained at the focus of attention, leading to facilitated retrieval. They found that mixed sets with a minority of emotional items were remembered better in an immediate serial recall test than those with a majority of emotional items. Their interpretation was that the enhanced attention to emotional items improved not only their own retrieval, but also the retrieval of neighboring neutral stimuli. The findings reviewed in this paragraph support the possibility that attention mediates the effect of emotion on memory, but this support is again inconclusive because attention was not measured explicitly.

Finally, a number of studies that did measure attention found that enhanced attention to emotional stimuli is accompanied by enhanced memory for them (Christianson, Loftus, Hoffman, \& Loftus, 1991; Mackay et al., 2004; Schmidt \& Saari, 2007; Talmi, Schimmack, et al., 2007). In an elegant and well-controlled study, Schmidt and Saari (2007) found that taboo words captured more attention than neutral words, measured via the latency to name their font color. Taboo words were also remembered better than neutral words in both blocked and mixed lists. The authors proposed that the attention effect accounted for the memory effect, but because the authors did not test formally whether the memory effect depended on the attention effect, and because EEM was not abolished in any of the conditions, the authors could not ascertain the causal role of attention. To reiterate, correlations between the effects of emotion on attention and memory are inconclusive because although enhanced attention to emotional stimuli could cause EEM, these two effects of emotional arousal could also occur in parallel.
So far we have reviewed evidence for the possibility that attention mediates the effect of emotion on memory. There is also evidence to the contrary. First, when capacity was not particularly limited, for example, when stimuli were presented slowly and/or when encoding was intentional, participants may have paid maximal - and hence, equivalent - attention to both emotional and to neutral stimulil; yet EEM was obtained (Kensinger \& Corkin, 2004; Kern et al., 2005; Talmi, Schimmack, et al., 2007). A stronger support is provided by studies that measure attention. In a previous study we measured attention and memory and tested the mediation effect formally. We found that the effect of negatively-valenced emotional arousal on memory was not mediated by attention, although attention did mediate the effect of positively-valenced emotional arousal on memory (Talmi, Schimmack, et al., 2007). Likewise, the number of eye fixations on a negative, emotionally arousing scene - a measurement which is considered to index visual attention - did not consistently predict memory for the scene (Christianson et al., 1991; Wessel, van der, \& Merckelbach, 2000). In fact, immediate EEM was obtained even when only one fixation per stimulus was permitted (Christianson et al., 1991). In summary, current evidence for the role of attention in EEM is mixed. Our hypothesis is that the reason it has been difficult to establish exactly what role attention plays in EEM is that its contribution interacts with that of other cognitive factors.

We have reviewed three factors that we hypothesize play a role in immediate EEM: organization, distinctiveness and attention. To decide which of them is necessary and sufficient to account for the effect, we need to know the conditions under which EEM is abolished. Our review shows that under full attention conditions EEM is only abolished when distinctiveness (Dewhurst \& Parry, 2000) or both distinctiveness and organization (Hadley \& Mackay, 2006, Experiment 1; Talmi \& Moscovitch, 2004; Talmi, Luk, et al., 2007) are controlled, but is obtained when these are not controlled (Kensinger \& Corkin, 2004; Kern et al., 2005; Talmi, Schimmack, et al., 2007). EEM is also abolished in situations which severely limit the contribution of any cognitive factor (Hadley \& Mackay, 2006; Sharot \& Phelps, 2004). This pattern emphasizes the importance of considering the three factors together in order to establish a comprehensive account for EEM.

The first goal of Experiment 1 was to reveal the contribution of attention to EEM by showing that EEM is obtained under divided attention even when distinctiveness and organization are controlled. Mediator analysis was to be employed to test formally whether EEM depends on enhanced attention to emotional stimuli even under these controlled conditions. The second goal of Experiment 1 was to demonstrate that all three factors - organization, distinctiveness and attention - provide a necessary and sufficient account for EEM. For this purpose Experiment 1 manipulated all three factors, with the aim to show that EEM only occurs when any one of them is not controlled.

Experiment 1 included a condition that matched the amount of attention allocated to blocked sets of emotional and equally-related neutral stimuli by employing a 
full-attention, intentional, slow-paced encoding phase. However, although we could demonstrate that organization and distinctiveness were equated across stimulus type (emotional and neutral) in this condition, we could not conclusively demonstrate that attention was also equated because this condition did not provide a measure of attention. Experiment 2 was designed to equate stimulus types on all three factors whilst providing a direct measure of attention using a divided-attention paradigm.

Together, the two experiments reported here were designed to examine the conditions in which EEM is abolished in order to develop and establish the cognitive account of immediate EEM.

\section{Experiment 1}

The first goal of Experiment 1 was to decide whether attention plays a role in immediate EEM when emotional and neutral items are equally distinct and equally interrelated. Primary distinctiveness was manipulated by presenting one group of participants with three blocked picture sets for encoding and immediate recall: one with negative pictures, one with related-neutral pictures (matched with the negative pictures on semantic relatedness) and one with random-neutral pictures (with lower relatedness than the other two sets). The other group was presented with three mixed sets, which included both emotional and neutral pictures (as in Talmi, Schimmack, et al., 2007). Primary distinctiveness was therefore controlled for the former, but not the latter, group.

The first dependent variable in this experiment was memory for the pictures. We measured memory using free recall, a measure thought to be most sensitive to the effects of emotion on memory (Dolan, 2002), because it is a measure of memory for gist, which is more strongly influenced by emotion than memory for detail (Adolphs, Tranel, \& Buchanan, 2005). Which free recall had been previously used to examine picture memory (Bradley, Greenwald, Petry, \& Lang, 1992) but, by necessity, the conclusion of our study would be limited to gist memory and cannot be generalized to memory for details.

Participants encoded the pictures while simultaneously performing an auditory discrimination task (following Talmi, Schimmack, et al., 2007). Performance on the auditory task provided a measure of attention, which was the second dependent variable in this experiment. This measure was expected to reveal that participants paid more attention to the emotional stimuli under both set composition conditions. Consequently, if attention contributes to EEM, then EEM should be obtained here under both set composition conditions (obtaining EEM under mixed-set dividedattention conditions would replicate Talmi, Schimmack, et al., 2007). Notably, under mixed-set conditions both distinctiveness and attention could contribute to EEM. If their contribution is not perfectly interactive, then EEM should be larger in the mixed-set than in the blocked-set condition.

Finally, on their own the memory and attention data cannot demonstrate conclusively that the enhanced attention to emotional stimuli mediated the EEM effect; the effect of emotion on attention and memory could be independent of each other. Mediator analysis was employed to answer this question.

The stimuli used in the following experiments, which have been made available to other researchers (http:// personalpages.manchester.ac.uk/staff/deborah.talmi), were developed in a series of pilot studies and consisted of emotionally-arousing, negatively-valenced pictures, matched with neutral pictures on low-level visual features, image complexity and people presence. The negative pictures were additionally matched with a subset of the neutral pictures on semantic relatedness. Only negatively-valenced emotional stimuli were used here for practical reasons, namely the greater ease in inducing robust and consistent emotional arousal across a sample of university students. The conclusions of this study will thus be limited to negatively-valenced emotions. Valence is considered an important factor in emotional memory, its contribution has been reviewed and evaluated elsewhere (Kensinger, 2004).

A perfect match between emotional and neutral stimuli is notoriously difficult, and the challenge grows as more ecologically valid stimuli are used. The emotional stimuli used here are, for example, less familiar than the neutral stimuli. Yet the influence of familiarity on memory is likely through its ability to alter the inter-relatedness, distinctiveness, and attention allocated to stimuli. By controlling and manipulating all of these potential down-stream effects of familiarity the following experiments resolve the psychological consequences of differential familiarity without having to control the stimuli for this factor. Finally, although we cannot be certain that the stimuli used here are perfectly matched on all of the factors that influence attention and memory, our experiments are less vulnerable than others to stimulus confounds because they are concerned with differences between conditions that utilize the same stimulus sets.

\section{Methods}

\section{Participants}

Seventy-two undergraduate students from the University of Toronto participated in the study for course credit (53 females, mean age $19.26, S D=2.12$ ). None had neurological or psychiatric history. Three participants had outlier performance on the concurrent task (more than 3 times the inter-quartile range) and were replaced. Participants were randomly allocated to the full-attention blocked (FA-blocked), divided-attention blocked (DA-blocked), and divided-attention mixed (DA-mixed) conditions. Data from the FA-blocked condition were reported previously (Experiment 4, Talmi, Luk, et al., 2007) and are included here to provide a baseline for the effect of divided attention.

\section{Materials}

The experimental pictures included 15 negative, 15 related neutral (domestic scenes) and 15 random neutral pictures which were drawn from the International Affective Picture System (Lang, Bradley, \& Cuthbert, 2005) and from the internet. Participants viewed three sets of 15 pictures. 
The mixed sets included five items of each type (negative, related-neutral, random-neutral), and the blocked sets included all 15 items of a single type. Care was taken to ensure that pictures did not resemble each other too closely, e.g. there was only one picture of a gun, to avoid confusion in scoring free recall data. 12 pilot participants rated all picture pairs for relatedness on a 7-point Likert scale with ' 1 ' indicating 'not at all related' and 7 indicating 'extremely related'. The relatedness score for each picture was computed as the average rated relatedness of that picture with other pictures of the same type in the list to which it was allocated, and overall relatedness scores for the emotional and the neutral sets were computed across picture for each participant. The emotional and related-neutral pictures were equally inter-related, and more inter-related than the random-neutral pictures. The number of pictures that depicted people was similar in each of the picture types. 12 pilot participants rated pictures on the dimensions of image complexity and brightness using Likert scales and on the dimensions of emotional valence and arousal using the Self Assessment Manikin (Bradley \& Lang, 1994). Emotional pictures were more negatively-valenced and more arousing than the neutral pictures, but picture types did not differ on complexity or brightness. The average scores for the blocked sets are reported in Table 1 . For reliability and other statistical tests confirming these findings see a previous report which used the same pictures (Talmi, Luk, et al., 2007, Experiment 4).

The three practice lists were similar to the experimental lists and included novel negative, related neutral (domestic scenes) and random neutral pictures. Buffer pictures included eight pictures of each type. Buffers for mixed-DA were sampled without replacement from this pool but buffers for the blocked condition were only sampled from the appropriate type. The four training pictures were random neutral. No ratings for these pictures were obtained. The stimuli for the auditory discrimination task were 250 , 750, and $2250 \mathrm{~Hz}$ pure tones, respectively, with $1 \mathrm{~s}$ duration. Materials for the arithmetic task were problems of addition and/or subtraction of two single digits (e.g. “4 $45=")$

Table 1

Characteristics of stimuli used in Experiment 1.

\begin{tabular}{llll}
\hline & Emotional & $\begin{array}{l}\text { Related- } \\
\text { neutral }\end{array}$ & $\begin{array}{l}\text { Random- } \\
\text { neutral }\end{array}$ \\
\hline Arousal & $5.74(0.80)$ & $2.66(0.39)$ & $2.91(0.41)$ \\
Valence & $2.80(0.63)$ & $5.05(0.35)$ & $5.11(0.23)$ \\
Visual complexity & $5.29(0.84)$ & $5.11(0.74)$ & $5.20(1.10)$ \\
Brightness & $4.87(0.72)$ & $5.40(0.94)$ & $4.90(0.65)$ \\
Relatedness & $1.77(0.29)$ & $1.69(0.16)$ & $1.15(0.09)$ \\
People presence & $0.73(0.46)$ & $0.53(0.52)$ & $0.67(0.49)$ \\
\hline
\end{tabular}

Note. Pilot participants in Talmi, Luk, et al. (2007) rated pictures for arousal and valence using the SAM scale where "1" represents "least arousing"|"most negative", and 9 represents "most arousing"|"most positive". They rated pictures for visual complexity, brightness and relatedness on a 1-7 Likert scale with "1" representing a low value and 7 representing a high value. Pictures got a score of "1" if they depicted people and "0" if they did not, and the proportion was calculated. See text for more details. Values in the table represent the mean of pictures in the pure sets, with standard deviation in parenthesis.

\section{Procedure}

Participants took part in four tasks: an auditory task, a picture-encoding task according to the attention condition they were assigned to, an arithmetic task, and a picture recall task. Participants were trained using shorter versions of all tasks. Following each training task participants received feedback on their performance. The training on the auditory task was repeated until participants reached an $80 \%$ accuracy criterion.

The auditory task required participants to listen to a random sequence of tones presented through headphones, and to press one key with the index finger of their dominant hand when they heard the $750 \mathrm{~Hz}$ tone, and another key with the middle finger of their dominant hand when they heard the other two tones. Tones were presented for $1 \mathrm{~s}$ followed by $1 \mathrm{~s}$ of silence. Participants were asked to respond as quickly as they could but before the next tone sounded.

The FA picture encoding task required that participants passively view a set of 15 pictures presented centrally on the monitor. Each picture was presented for $2 \mathrm{~s}$ followed by a blank screen for $4 \mathrm{~s}$ to minimize carry-over effects. Picture order was randomized for each participant. Two buffer items were inserted before and after the experimental picture sets to control for primacy and recency effects. Buffer items were randomly allocated to each list, but in the pure list condition buffers always were of the same type as the experimental pictures (e.g. negative pictures served as buffers in the negative picture list; see materials section).

The DA picture encoding task required participants to perform the picture encoding task while simultaneously performing the auditory task. A tone was presented at picture onset (Stimulus Onset Asynchrony $(\mathrm{SOA})=0$ ), another one at picture offset $(S O A=2)$, and a third during the interstimulus interval $(\mathrm{SOA}=4)$. Participants were requested to devote $99 \%$ of their attention to the auditory task and use the remaining $1 \%$ of their attention to view the pictures. These numbers were used to communicate to participants the relative importance of the two tasks and create an ordinal relationship between the full and the divided attention conditions; we did not expect participants to be able to divide their attention exactly according to these percentages. The instructions stated: "Please allocate $99 \%$ of your attention to the tone task. This is critical for the success of the experiment, so please do your best to manage your attention in this way. This means that you would listen and respond to the tones as quickly and accurately as you can. Use the remaining $1 \%$ of your attention to commit the pictures to memory."

The arithmetic task was performed during a 1-min retention interval which followed the picture encoding task. This task was designed to displace encoded pictures from working memory, by engaging visual processing, decision making, and silent verbalization processes, and had been successfully used for this purpose (Talmi, Grady, Goshen-Gottstein, \& Moscovitch, 2005). The task required participants to compute the value of the left and the right arithmetic problem and select the problem with the higher value by pressing the corresponding arrow key. When they made their selection the next two problems appeared until 
1 min had elapsed. This task always followed the picture encoding task.

The free recall task immediately followed the arithmetic task and required participants to write down a brief description of the pictures they saw in the preceding picture encoding task in any order. Participants were given $3 \mathrm{~min}$ for this. At the end of the free recall period, the next set of pictures was presented. The sequence of picture encoding, arithmetic, and free recall tasks repeated once during initial practice, with a short picture set. It was then repeated three times with the full-length practice picture sets. The auditory task was then performed alone, using 171 tones. Finally, the sequence was repeated again with the three experimental lists.

Because participants in the DA condition performed the auditory-discrimination task alone before they performed it under divided attention (DA), any decline in performance in the latter condition would demonstrate a cost due to division of attention that was greater than the benefits due to the extra practice.

A cycle of these three tasks - picture encoding, arithmetic and the picture recall - repeated three times, once with each of the three practice picture sets (negative, relatedneutral, random-neutral, or three mixed lists). The auditory task was then performed alone. The cycle of encoding, arithmetic and recall then repeated three more times, once with each of the three experimental picture sets (negative, related-neutral, random-neutral, or three mixed lists). Thus, in the pure list condition participants encoded only a single list of practice pictures and a single list of experimental pictures of each type.

At the end of the experiment participants were asked to write down what themes they noticed amongst the studied pictures. The results reported below use a significance threshold of $p<.05$ and report the 95\% confidence interval for all theoretically-important comparisons.

\section{Results}

\section{Picture memory}

We followed the procedure employed by Bradley et al. (1992) for scoring picture recall. The recall sheets were scored on the basis of the participant's description of the picture and correct recall score meant that the rater believed she could clearly link the description of a particular picture to a studied picture. Free recall data were scored by two raters, the experimenter and a second rater blind to the purpose of the study. Disagreements were minimal, with a correlation of $r=.99, p<.001$ between the two raters, and were resolved through discussion. Descriptions that could not be assigned to any of the studied pictures were classified as intrusions. These represented an average of $1.09 \%$ of the total recall in FA, $2.24 \%$ in DA-blocked and 9.21\% in DA-mixed.

Fig. 1 shows that EEM was obtained under DA, where it was larger in mixed-DA than blocked-DA, but not under FA, where memory for related-neutral pictures equaled memory for negative pictures. Neither attention, nor set composition conditions influenced memory for negative pictures, but memory for neutral pictures was impaired under DA, and more so under mixed-DA than blocked-DA.

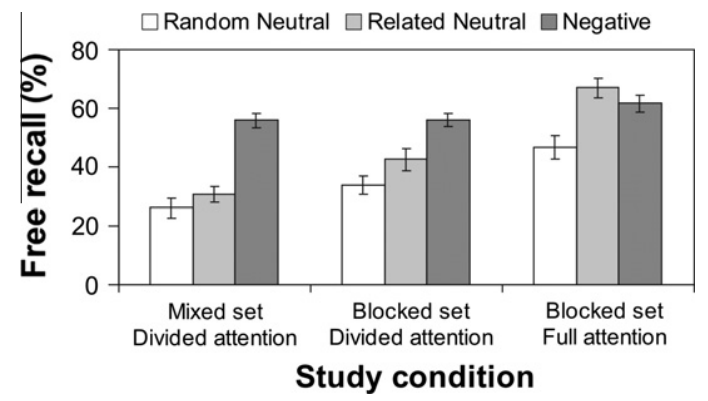

Fig. 1. Mean free recall (in percent) in Experiment 1 as a function of picture type and study condition. Error bars represent standard errors.

Recall was analyzed as a function of picture type (negative, related-neutral, random-neutral) with repeatedmeasures ANOVA, using study condition (FA-blocked, DAblocked, DA-mixed) as a between-subject factor. The main effects of picture type, $F(2138)=59.73, p<.001$, partial $\eta^{2}=.46$, and study condition, $F(2,69)=2.89, p<.001$, partial $\eta^{2}=.37$, were significant. Their interaction was also significant, $F(4138)=9.57, p<.001$, partial $\eta^{2}=.22$, due to the fact that study condition had a more pronounced influence on neutral than on emotional memory.

We followed up on this significant interaction by analyzing the equally-related emotional and related-neutral pictures under the two DA conditions. There was a significant effect of picture type, $F(1,46)=61.60, p<.05$, partial $\eta^{2}=.57$, and a significant interaction between picture type and set composition, $F(1,46)=5.54, p<.05$, partial $\eta^{2}=.11$.

Bonferonni-corrected $t$-tests were carried out to clarify which conditions obtained a significant EEM. Under FA negative pictures, $M=61.66, S D=13.80$, were recalled better than random-neutral pictures, $M=46.67, S D=19.26$, $t(23)=4.22, p<.001$, Cohen's $d=.90, C_{95}=7.65,22.34$, but slightly worse than related-neutral pictures, $M=66.94, S D=16.74, t(23)=1.68, p>.10$, Cohen's $d=.34$, $\mathrm{Cl}_{95}=-11.76,1.21$, suggesting that EEM was only obtained when organization was not controlled. Examining the mean free recall across conditions demonstrates that this null effect was not due to ceiling effect in recall. Under DA negative pictures (DA-blocked: $M=56.11, S D=10.39$; DA-mixed: $M=55.83, S D=12.56)$ were recalled better than both sets of neutral pictures. Importantly, they were recalled better than related-neutral ones in both DAblocked, $M=42.5, S D=17.89, t(23)=4.07, p<.001$, Cohen's $d=0.93, \mathrm{Cl}_{95}=6.69,20.53$, and DA-mixed, $M=30.55$, $S D=13.61, \quad t(23)=6.91, \quad p<.001, \quad$ Cohen's $d=1.93$, $\mathrm{Cl}_{95}=17.71,32.84$.

\section{Auditory task}

Fig. 2 shows that viewing negative pictures impaired performance on the auditory task, a decline which was reflected in both latency and accuracy measures. Despite the impairment in performance when participants viewed negative pictures, there were no significant interactions with set composition.

Participants in the DA condition performed the auditory task better when it was presented alone than concurrently with picture encoding: accuracy dropped from $96 \%$ 

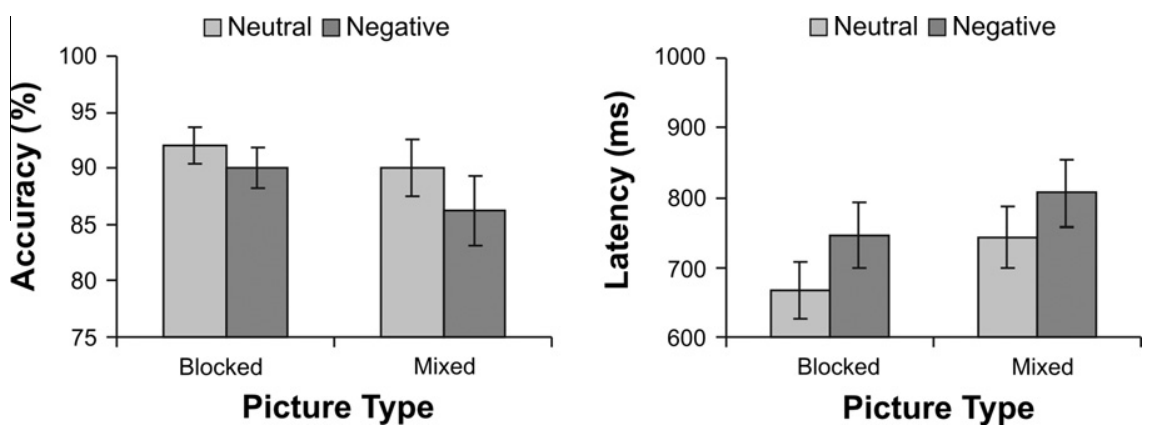

Fig. 2. Auditory task performance in Experiment 1. Accuracy (left) and latency (right) in the auditory task as a function of picture type (negative, related neutral) and set composition (blocked, mixed), averaged over SOAs. Error bars represent standard errors.

$(S D=2.92 \%)$ under $\mathrm{FA}$ to $90 \%(S D=8.77 \%)$ under $\mathrm{DA}$, $F(1,47)=28.65, p<.001$, partial $\eta^{2}=.38$, and latency increased from $497 \mathrm{~ms}(S D=139 \mathrm{~ms})$ under $F A$ to $712 \mathrm{~ms}$ $(S D=192 \mathrm{~ms})$ under DA, $F(1,47)=155.26, p<.001$, partial $\eta^{2}=.77$.

Latency and accuracy scores were analyzed separately with a 2 (set composition: DA-blocked, DA-mixed) by 3 (picture type: negative, related-neutral, random-neutral) by 3 (SOA: $0,2,4)$ repeated-measures ANOVAs with set composition (blocked, mixed) as a between-subject factor. The latency data exhibited significant main effects of picture type, $F(2,92)=15.90, p<.001$, partial $\eta^{2}=.26$, and SOA, $F(2,92)=10.68, p<.001$, partial $\eta^{2}=.19$. There accuracy data also exhibited significant main effects of picture type, $F(2,92)=6.51, p<.005$, partial $\eta^{2}=.12$, and SOA, $F(2,92)=52.27, p<.001$, partial $\eta^{2}=.53$.

Because the related-neutral pictures provide a better comparison for the emotional ones, the analyses were repeated without the random-neutral pictures. As predicted, latency was longer when participants viewed negative pictures, $M=777 \mathrm{~ms}, S D=31 \mathrm{~ms}$, than related-neutral pictures, $\quad M=704 \mathrm{~ms}, \quad S D=27.88 \mathrm{~ms}, \quad F(1,46)=20.33$, $p<.001$, partial $\eta^{2}=31, \mathrm{Cl}_{95}=40 \mathrm{~ms}, 104 \mathrm{~ms}$. Latency was also influenced by SOA, $F(2,92)=10.45, p<.001$, partial $\eta^{2}=.18$; there was a non-significant trend for an interaction between picture type and SOA, $F(2,94)=2.88, p=.06$, partial $\eta^{2}=.06$.

Also as predicted, accuracy was impaired when participants viewed negative pictures, $M=88.19 \%, S D=1.48 \%$, relative to related neutral pictures, $M=91.20 \%, S D=1.23 \%$, $F(1,46)=13.36, p=.001$, partial $\eta^{2}=.22, C l_{95}=-4.66 \%$, $1.35 \%$. Accuracy was also influenced by SOA, $F(2,92)=34.77, p<.001$, partial $\eta^{2}=.43$; the effects of picture type and SOA did not interact, $p>.10$. Crucially, there were no significant effects of set composition on either latency or accuracy, $p>.10$.

\section{Mediator analysis}

The above results demonstrate that in line with predictions, picture type influenced both attention and memory. These results, on the own, cannot tell us whether these two effects of emotion were related to each other or occurred in parallel.

Mediatory analysis was employed to answer this question. The following analyses was carried out across pictures instead of across subjects, following Talmi, Schimmack, et al. (2007). To avoid having to consider the effect of relatedness on the mediation effect random-neutral pictures were excluded from this analysis.

The average latency and accuracy on the auditory discrimination task when it was performed alone was computed across participants. In the DA conditions the three latency and three accuracy measurements obtained for each participant in each SOA were averaged across participants and SOA, but separately for the DA-mixed and DAblocked conditions. The result was one accuracy and one latency score per picture in each of the two DA conditions Attention-cost scores were then computed per picture, seperately for each DA condition. Accuracy cost scores were computed by subtracting the accuracy with which the auditory task was performed under DA from the accuracy with which it was performed alone. Latency cost scores were computed by subtracting the latency with which the auditory task was performed alone from the latency with which it was performed under DA. This procedure meant that higher attention cost scores reflected a larger cost from concurrent picture encoding. Notably, subtracting the baseline (auditory task alone) separately in the DA-blocked and DA-mixed condition ensured that the comparisons across these conditions would not be contaminated by potential group differences. We computed an arousal score per picture by averaging across the emotional arousal ratings provided by pilot participants.

As expected, in both set-composition conditions arousal significantly correlated with recall and with attention, and attention and recall were correlated with each other (see Table 2). To check for unique contributions of arousal and

Table 2

Correlations between arousal, latency and accuracy cost, and free recall in Experiment 1.

\begin{tabular}{llll}
\hline & Recall & Latency cost & Accuracy cost \\
\hline DA-mixed & & & \\
Arousal & $.70^{* * *}$ & $.64^{* * *}$ & $.53^{* * *}$ \\
Recall & & $.45^{* *}$ & $.51^{* *}$ \\
DA-blocked & & \\
Arousal & $.47^{* *}$ & $.80^{* * *}$ & $.35^{\dagger}$ \\
Recall & & $.60^{* * *}$ & $\mathrm{n} . \mathrm{s}$ \\
\hline${ }^{* * *} p<.001$. & & \\
$*^{* *} p<.01$. & & \\
$p<.05$. & & \\
${ }^{*} p>.05$ and $p<.10$. & &
\end{tabular}


attention to memory a multiple regression analysis was carried out with recall as the dependent measure and the predictors set composition (dummy coded), arousal, latency cost, accuracy cost, and the interaction between set composition and each of the other factors. The model was significant overall, $F(7,52)=6.66, p<.001$, and explained $47 \%$ of the variance. This analysis revealed main effects of arousal $(\beta=.7, t=3.59, p=.001)$ as well as a significant interaction between latency cost and set composition $(\beta=.1 .36, t=2.17, p<.05)$ and between arousal and set composition $(\beta=-1.03, t=-2.30, p<.05)$. Because these interactions suggested that the contributions of arousal and attention to memory depend on set composition, separate multiple regression analyses were conducted for the DA-mixed and DA-blocked conditions using arousal, latency cost, and accuracy cost as predictors. Fig. 3 depicts the results of these analyses.

In the analysis of the DA-blocked condition only latency cost $(\beta=.69, p<.05)$ predicted recall. Neither arousal, nor accuracy cost, had a significant contribution (arousal $\beta=-.01, p>.10$, accuracy cost $\beta=-.19, p>.10$ ). Given the correlation pattern in Table 2 , the non-significant effect of attention on recall in this condition when arousal was also included in the regression analysis clearly suggest that attention fully mediates the effect of arousal on memory (Baron \& Kenny, 1986).

By contrast, analysis of the DA-mixed condition revealed that only arousal significantly predicted recall, $(\beta=.62, p<.01)$, but latency and accuracy costs did not have a significant contribution (latency cost $\beta=-.06$, $p>.10$; accuracy cost $\beta=.21, p>.10$ ), replicating Talmi, Schimmack, et al., 2007). Taken together, these results suggest that attention only mediated the effect of arousal on recall under DA-blocked, but not under DA-mixed.

This interpretation can only be drawn tentatively because a comparison of methods to assess mediation effects (MacKinnon, Lockwood, Hoffman, West, \& Sheets, 2002)

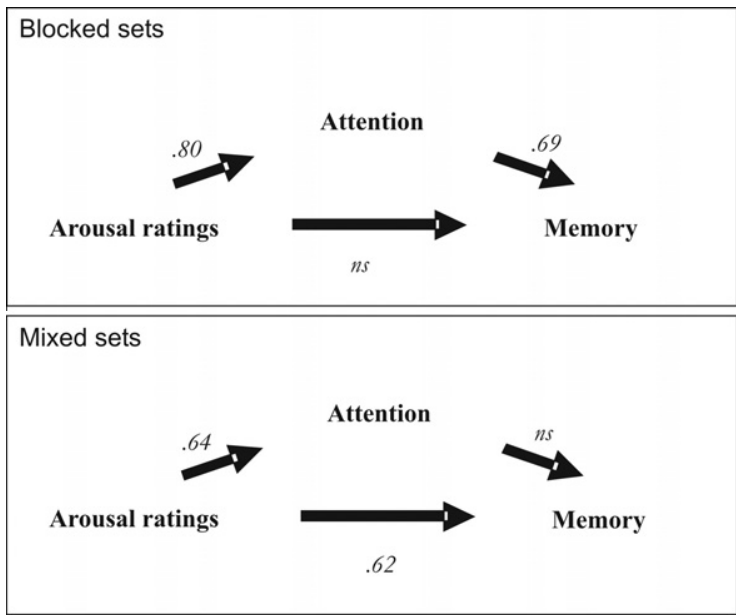

Fig. 3. Mediator analysis for results from Experiment 1 shows that the predictors of immediate memory differ as a function of set composition. Attention predicted memory when picture type was blocked (top panel), but arousal predicted memory when pictures were studied in mixed sets (bottom panel), a condition in which emotional pictures had higher primary distinctiveness than neutral ones. revealed that multiple regression suffers from limited power to detect mediation effects and that the Sobel test (Baron \& Kenny, 1986) is a more direct and more powerful method of measuring the significance of indirect effects. The bootstrapped ratio (Preacher \& Hayes, 2004), is comparable to the Sobel test but is specialized for use with relatively small sample sizes. The bootstrapped ratio is a nonparametric test of the hypothesis that the indirect effect is significant and is preferable because it does not assume normal and symmetrical distribution of indirect effects, an assumption which is often violated in small sample sizes (Preacher \& Hayes, 2004). The bootstapped ratio makes no assumption about the shape of the distribution of this effect and instead uses the sampling distribution to derive a confidence interval. Here we used 1000 bootstraps and a 95\% confidence interval. The bootstrapped ratio was significant for the DA-blocked condition equate stimulus types on all three factors whilst providing, $p<.05$, but not the DA-mixed condition, $p>.10$. This result confirms the interpretation of the multiple regression analysis reported above.

Finally, to illuminate the causes of EEM the same multiple regression analyses were conducted for negative and related-neutral pictures separately. The multiple regression models were significant for negative [DA-mixed: $F(3,11)=4.88, \quad p<.05 ; \quad$ DA-blocked: $\quad F(3,11)=11.45$, $p=.001]$, but not related-neutral pictures. For negative pictures, arousal predicted memory under DA-mixed $(\beta=.91$, $t=3.21, p<.01)$ but not DA-blocked $(\beta=.05, t<1)$. By contrast, latency cost predicted memory under DA-blocked $(\beta=.85, t=3.09, p=.01)$ but not DA-mixed $(\beta=-.29$, $t<1)$. These results show that the interesting difference between the memory predictors in each set-composition condition stemmed from differences between the predictors of memory for negative pictures.

\section{Report of themes}

Participants received two scores, one for reporting the negative theme and one for reporting the related-neutral theme. Scores were binary and liberal, so that participants received a ' 1 ' for mentioning themes such as 'violence' or 'doing things around the house'. All participants except one (under $\mathrm{DA}=$ mixed) reported the negative theme but report of related-neutral theme was less frequent: $30 \%$ under DA-mixed, 71\% under DA-blocked, and 63\% under FAblocked. Memory results, however, were the same for participants who were or were not aware of the relatedneutral theme. An ANOVA with study condition and neutral theme report as between-subject factors and picture type as within-subject factors revealed no significant main effect or interactions with theme report.

\section{Discussion}

When organization and distinctiveness were controlled EEM depended on the attention capacity available to participants. Under DA-blocked emotional stimuli captured more attention than neutral stimuli, as predicted, and EEM was obtained. When capacity was less restricted (FA-blocked), however, there was no significant EEM. These null results under FA-blocked are robust, as similar 
results were obtained in three experiments by Talmi, Luk, et al. (2007). On their own, these data suggest but do not conclusively demonstrate that enhanced attention to individual emotional stimuli caused EEM. Crucially, data from the mediator analysis clarified that attention fully mediated the effect of emotion on memory, but only when organization and distinctiveness were controlled.

There were similarities in the results from the two divided attention conditions, DA-mixed and DA-blocked. Under both conditions emotional stimuli captured greater attention resources than neutral stimuli. This effect was statistically equivalent under the two conditions. Although the latter is a null result, it suggests that the influence of emotion on attention is not due to the higher primary distinctiveness of emotional stimuli. What, then, underlies the ability of these stimuli to capture attention? The emotional stimuli were more arousing, more negative, and higher in secondary distinctiveness than the neutral ones, and any of these factors could account for their ability to capture attention. Previous work, however, suggests that arousal is the key factor (Schimmack, 2005). Taken together, the finding that emotional items were attended equally under the two DA conditions but EEM was greater under DA-mixed than DA-blocked suggests that the higher primary distinctiveness of emotional pictures under DAmixed contributes to EEM over and above the effect of enhanced attention to these stimuli.

Results from the mediator analysis illuminate the interplay between the influences of distinctiveness and attention on EEM. Our interpretation relies on the assumption that the arousal ratings measure not only emotional arousal, but also distinctiveness. There are therefore two potential reasons for the finding that arousal ratings predicted memory under DA-mixed. We contend that distinctiveness must have played a role in this effect because a pure arousal effect should have persisted across set composition conditions, yet arousal ratings did not predict memory even partially under DA-blocked. One possibility is that the arousal associated with the emotional stimuli draws extra attention to them, and this extra attention enables participants to elaborate on their feelings, making the emotional pictures more distinct at retrieval, and leading to enhanced memory under DA-blocked. Such elaboration may help memory less under DA-mixed because at retrieval all emotional items are more distinct than the neutral ones. Note that accounts that rely on preferential maintenance of emotional stimuli in the focus of attention (Majerus \& D'Argembeau, 2011) do not apply to our study because two buffer items and a distractor task were interpolated between encoding and retrieval.

A surprising finding in Experiment 1 was that participants reported the theme of the neutral pictures less frequently than that of emotional pictures. Theme report appeared to be influenced more by the number of pictures of each type in the list and their temporal contiguity (Hunt \& Seta, 1984), rather than by attention resources, because theme report was similarly high in the full and dividedattention blocked-set conditions, but lower in the DAmixed condition where there were only a few pictures of each type. One possibility is that although participants utilized the semantic links between items, evident in the superior memory for related-than random-neutral items, they may have not classified stimuli to themes during the experiment itself but did so only when they were explicitly asked to report the theme at the end. This interpretation is supported by the low correlation between theme report and memory. Another possibility is that the emotional theme may have been more accessible because information about disturbing images was provided in the information sheet and consent form. To improve the match between emotional and neutral stimuli, Experiment 2 replicated the divided-attention conditions, where the report of emotional and neutral themes was least well-matched, but participants were informed of upcoming themes before encoding began.

Experiment 1 also provided further evidence that organization contributed to EEM. This was evident in larger EEM when organization was not controlled, in the comparison of negative and related-neutral vs. random-neutral pictures. To summarize, Experiment 1 succeeded in its two goals. It showed that attention contributes to EEM and demonstrated that organization, distinctiveness, and attention are all necessary to account for EEM. The finding that memory for negative and related-neutral pictures was equivalent under FA-blocked, a condition where all three factors were best matched across stimulus types, suggests that these three factors also provide a sufficient to account of EEM. However, the FA-blocked condition did not provide a direct measure of attention. The main goal of Experiment 2 was therefore to support the hypothesis that the three factors provide a necessary and sufficient account for EEM by matching emotional and neutral stimuli on all three factors, but this time, measure attention directly.

Showing that EEM can be abolished under divided attention is important because these conditions do not only reduce the amount of attention to stimuli but may also change encoding qualitatively, for example by modulating the influence of organization and distinctiveness on memory (Craik \& Kester, 1999; Mitchell \& Hunt, 1989). Encoding emotional and neutral stimuli may therefore be processed especially disparately under divided attention, leading to EEM.

\section{Experiment 2}

Equating the level of attention to emotional and neutral pictures is challenging because emotional items capture participants' attention involuntarily, even when this emotional engagement hurts their experimentally assigned goals (Vuilleumier, 2005). This is evident in results from Experiment 1 because participants' attention was directed towards picture encoding even though their assigned task was to maximize performance on the auditory task.

Equating attention to emotional and neutral stimuli may be achieved by providing participants with a lot of encoding resources or depleting encoding resources to a minimum. Unfortunately, neither approach provides a behavioral measure of attention, and without such a measure, it is impossible to verify the success in equating attention to the two stimulus types. Experiment 2 addresses this challenge using a procedure developed by 
Talmi, Schimmack, et al. (2007). This procedure equates the amount of attention allocated to emotional and neutral items while also providing a measure of attention.

In Experiment 2 participants encoded blocked sets of negative and related-neutral pictures under divided attention. The amount of attention participants allocated to stimuli was measured as in Experiment 1, by using performance on a concurrent auditory task. We varied the amount of attention participants allocated to pictures by instructing them to allocate either $1 \%$ or $50 \%$ of their attention to the pictures. Participants encoded one emotional set and one neutral set under instructions to place $1 \%$ of their attention on the pictures (99/1 condition). They also encoded one emotional and one neutral set under instructions to place $50 \%$ of their attention on the pictures $(50 / 50$ condition). In both conditions they were asked to allocate the remainder of their attention to the auditory task. The experiment thus conforms to a 2 (instructions: 50/50, 99/ 1 ) by 2 (picture type: emotional, neutral) design. Participants were not expected to be able to utilize these exact percentages $(1 \%, 50 \%, 99 \%)$, but, on the basis of previous studies (Craik et al., 1996) were expected to be able to divide their attention in an ordinal manner, and attend the pictures more in the 50/50 condition than in the 99/1 condition.

The selection of 50/50 and 99/1 percentages was based on previous results (Talmi, Schimmack, et al., 2007) where the analysis of concurrent task performance revealed that participants' attention to negative pictures encoded under the 99/1 condition, and their attention to neutral pictures encoded under the 50/50 condition was equivalent. The comparison between these conditions is referred to as the 'critical comparison' in Experiment 2. Note that the predicted significant main effects of instructions and picture type in the analysis of the concurrent task could be used to argue that a null effect in the critical comparison is not due to insufficient power.

The two critical conditions were matched for organization and distinctiveness because they included blocked sets of equally-related stimuli. If the critical comparison would reveal that they were also matched for attention, a memory advantage for the negative pictures would suggest that something else, beyond organization, distinctiveness, and attention, underlies immediate EEM. A null result, however, would support the cognitive account of immediate EEM and would show that organization, distinctiveness and attention provide a sufficient account for this effect. Again, the predicted main effects in the analysis of memory were of less interest, but could be used to argue against a power-dependent interpretation of the results of this critical comparison.

As we mentioned above, we have already used this procedure in a study that employed mixed sets of negative and neutral pictures (Talmi, Schimmack, et al., 2007). That study obtained EEM in the critical comparison, despite picture sets being matched on attention and organization. EEM in that study could, however, have been due to differential primary distinctiveness because that study only used mixed sets. In Experiment 2 we eliminated this confound by using blocked sets of negative and neutral stimuli.
To summarize, the critical comparison in Experiment 2 was between negative and neutral stimulus sets that were equally inter-related, had equal primary distinctiveness, and were predicted to also be equally attended. To our knowledge, this is the first study that matched emotional and neutral stimuli simultaneously on all three factors. If these three factors are necessary and sufficient for immediate EEM, EEM would be found within each one of the instruction conditions $(50 / 50,99 / 1)$ but not in the critical comparison. Experiment 2 also provided a replication for Experiment 1 using a modified stimulus set.

\section{Methods}

\section{Participants}

Twenty-four second-year students in the University of Manchester, UK, took part in the study as part of the experiment credit scheme (three males, mean age 19.47, $S D=.99$ ). None had neurological or psychiatric history. The study was approved by the School of Psychological Science ethics committee. Considering the effect size from the blocked-DA condition in Experiment 1, which most resembled Experiment 2, power calculations (Faul, Erdfelder, Lang, \& Buchner, 2007) revealed that a sample size of 22 participants was would have a power of .95 to detect a difference between memory for related and negative pictures.

\section{Materials}

Because in this experiment attention instructions were manipulated within-subject, each participant needed to encode two sets of 15 pictures of each type, doubling the number of stimuli relative to Experiment 1 . A new set of stimuli was therefore created and validated. Neutral pictures depicting domestic scenes and negative, emotionally-arousing pictures, 42 of each type, were drawn from the IAPS (Lang et al., 2005) and from the internet, and resized to $280 \times 210$ pixels. All pictures depicted people. Pictures were rated for relatedness using the procedure described above by 14 students at the University of Alberta, Canada (11 females) who were given course credits. Relatedness ratings were reliable (Cronbach's alpha >.8). Thirty negative and 30 neutral pictures were selected from this pool for use in Experiment 2 and divided into two formats, each with 15 pictures of each type. For each participant, a relatedness score was computed for each picture by averaging the relatedness ratings the participant gave the picture when it was paired with all other 14 pictures of its type in the same format; these scores were then averaged across pictures in each of the two formats. Average relatedness scores were 3.86 and 3.87 for negative pictures in formats 1 and 2, respectively, and 3.49 and 3.51 for neutral pictures in formats 1 and 2, respectively. These scores were analyzed with a 2 (format: 1,2 ) by 2 (picture type: negative, related-neutral) repeated-measures ANOVA. None of the effects were significant [picture type: $F(1,13)=1.99, p>.10$, partial $\eta^{2}=.13, \mathrm{Cl}_{95}=-.19, .93$; Format, $F<1$, partial $\eta^{2}<.02$; the interaction: $F<1$, partial $\left.\eta^{2}<.01\right]$. This experiment did not include random neutral pictures.

The 60 experimental pictures were rated for emotionality by a group of 44 University of Manchester students 
using a computerized SAM scale (Bradley et al). Valence and Arousal scores are described in Table 3. These scores were analyzed with two format by picture type repeatedmeasures ANOVAs. The negative pictures were more negative than the neutral ones, $F(1,43)=262.22, p<.001$, partial $\eta^{2}=.86, \mathrm{Cl}_{95}=-3.04,-2.37$. The main effect of format, $F(1,43)=3, p=.06$, partial $\eta^{2}=.08$, and its interaction with picture type, $F<1$, were not significant. The negative pictures were also more arousing than the neutral ones, $F(1,43)=85.10, p<.001$, partial $\eta^{2}=.66, \mathrm{Cl}_{95}=2.17$, 3.83 , but the effect of format, $F<1$ and the interaction between format and picture type, $F(1,43)=3.39, p=.07$, partial $\eta^{2}=.07$, were not significant. The experimenter also selected 15 unrated neutral pictures of domestic scenes and 15 negative pictures to be used for practice, and four pictures of landscapes to be used for training.

\section{Procedure}

The procedure resembled the procedure of Experiment 1, DA-blocked condition, with the following exceptions. One picture format, including an emotional and a neutral picture set, were studied under 99/1 emphasis, a condition in which participants were asked to allocate $99 \%$ of their attention to the auditory task and only allocate $1 \%$ of their attention to the pictures. The other picture format was studied under 50/50 emphasis, a condition in which participants were asked to allocate $50 \%$ of their attention to the auditory task and $50 \%$ of their attention to the pictures. The initial training list was studied under 75/25 emphasis, a condition in which participants were asked to allocate $75 \%$ percent of their attention to the auditory task and $25 \%$ of their attention to the pictures. The instructions to participants were identical to those used in the DA picture

Table 3

Valence and arousal ratings for the stimulus set used in Experiment 2.

\begin{tabular}{llllll}
\hline & \multicolumn{2}{l}{ Valence } & & Arousal & \\
\cline { 2 - 3 } \cline { 5 - 6 } & Neutral & Negative & & Neutral & Negative \\
\hline Set 1 & 5.12 & 2.47 & & 2.05 & 5.13 \\
Set 2 & 5.43 & 2.67 & & 2.38 & 4.85 \\
Average & 5.27 & 2.57 & & 2.22 & 4.99 \\
Set 1 & 0.55 & 0.86 & & 1.10 & 1.62 \\
Set 2 & 0.95 & 1.10 & & 1.24 & 2.01 \\
Average & 0.75 & 0.98 & 1.17 & 1.82 \\
\hline
\end{tabular}

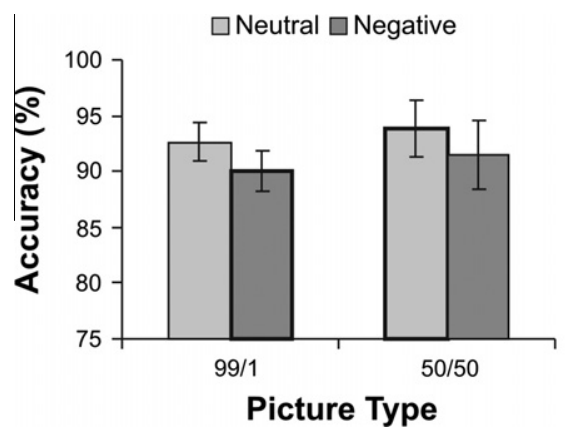

encoding task in Experiment 1, but the stated percentages matched the emphasis condition. Finally, because in Experiment 1 participants reported the theme of the relatedneutral pictures less frequently than that of the emotional pictures, they were now informed of the theme of each upcoming set of pictures.

\section{Results}

\section{Auditory task}

Two participants had one statistically outlying score (more than three times the inter-quartile range) on the auditory task; they are included in the following analysis but results do not change if they are excluded. Fig. 4 shows that viewing negative pictures impaired performance on the auditory task according to both latency and accuracy measures. Latency and accuracy scores were analyzed separately with a 2 (picture type: negative, related-neutral) by 2 (instructions: 99/1, 50/50) by 3 (SOA: 0, 2, 4) repeatedmeasures ANOVAs. As predicted, latency was longer when participants viewed negative pictures, $M=872, S D=23$, than related-neutral pictures, $M=807.43, S D=21$, $F(1,23)=15.60, p=.001$, partial $\eta^{2}=.40, \mathrm{Cl}_{95}=31,99$. As predicted, latency was longer when participants devoted $50 \%, M=878, S D=23$, instead of $99 \%, M=801, S D=23$ of their attention to the tones, $F(1,23)=15.36, p=.001$, partial $\eta^{2}=.40, \mathrm{Cl}_{95}=36,118$. As predicted, accuracy was impaired when participants viewed negative pictures, $M=93.2 \%, S D=1 \%$, relative to related-neutral pictures, $M=90.8 \%, \quad S D=1.4 \%, \quad F(1,23)=8.58, \quad p<.01, \quad$ partial $\eta^{2}=.27, \mathrm{Cl}_{95}=-4.2 \%,-.7 \%$. Accuracy was also influenced by SOA, $F(2,46)=7.68, p<.01$, partial $\eta^{2}=.25$.

The two critical conditions, negative pictures under 99/ 1 and neutral pictures under 50/50, were compared using two separate 2 (picture type: negative, related neutral) $\times 3$ (SOA: 0, 2, 4) repeated measure ANOVAs. For latency, neither the main effect of picture type, $F<1$, partial $\eta^{2}<.01$, $\mathrm{Cl}_{95}=-44,69.50$, nor its interaction with SOA, $F<1$, partial $\eta^{2}=.03$, were significant. The main effect of SOA was also not significant, $F<1$. For accuracy, neither the main effect of picture type, $F(1,23)=1.34, p=.26$, partial $\eta^{2}=.06$, $\mathrm{Cl}_{95}=-0.9 \%, 3.3 \%$, nor its interaction with SOA, $F<1$, partial $\eta^{2}=.01$, were significant. The main effect of SOA was significant, $F(2,46)=8.24, p<.01$, partial $\eta^{2}=.26$. The absence of any significant differences in concurrent task

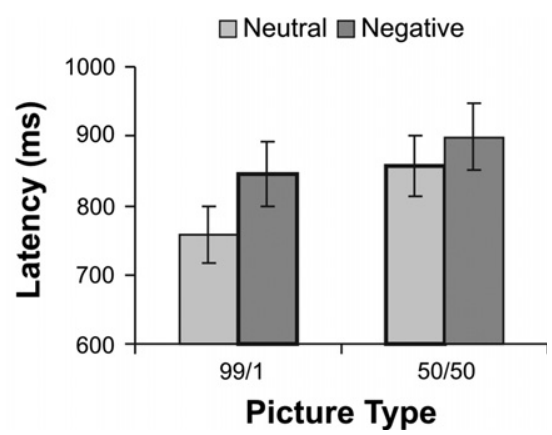

Fig. 4. Auditory task performance in Experiment 1. Accuracy (left) and latency (right) in the auditory task as a function of picture type (negative, related neutral) and instructions $(99 / 1,50 / 50)$, averaged over SOAs. Error bars represent standard errors. 
performance between these two critical conditions conforms our previous results (Talmi, Schimmack, et al., 2007) and demonstrates that participants devoted equal amounts of attention to emotional pictures encoded under 99/1 conditions and neutral pictures encoded under 50/50 conditions.

Taken together, the fact that the experiment had sufficient power to detect the effect of picture type within instruction condition, the substantial magnitude of this effect, and the small magnitude of the non-significant effect of picture type across instruction conditions (the critical comparison) suggest that the difference between the amount of attention allocated to negative and neutral pictures in these two conditions was negligible, and, at the very least, that attention to both picture types was much better matched in this critical comparison than within each instruction condition.

\section{Picture memory}

Free recall was initially scored by two experimenters and disagreement was resolved by discussion. There was a high correlation between their agreed score and those of a third rater, who was blind to the purpose of the study, $r=.94, p<.05$. Descriptions that could not be assigned to any of the studied pictures were classified as intrusions, an average of $24.66 \%$ of total recall output. This higher percentage relative to the DA-blocked condition in Experiment 1 likely reflects the fact that participants encoded two sets instead of a single set of stimuli of each type. Given this higher number, the percentage of intrusions relative to total recall was analyzed as a function of picture type and instructions $(99 / 1,50 / 50)$ with a repeated-measures ANOVA. There were no significant effects.

Fig. 5 shows that participants recalled more emotional than neutral pictures under both attention allocation conditions but there was no advantage for emotional pictures when both picture types were equally attended. Picture memory was analyzed as a function of picture type and instructions $(99 / 1,50 / 50)$ with a repeated-measures ANOVA. As predicted, negative pictures, $M=6.12$, $S D=.301$, were recalled more frequently than related-

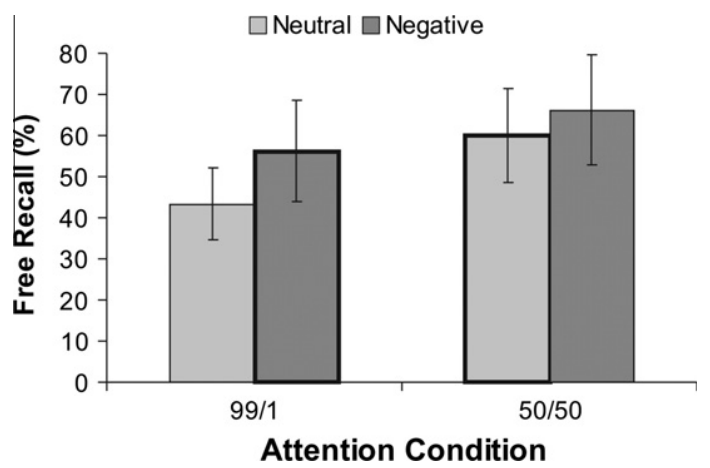

Fig. 5. Mean free recall (in percent) in Experiment 2 as a function of picture type and instructions (99/1, 50/50). The bold frame around the two middle bars represents conditions in which participants attended neutral and negative pictures equally. Error bars represent standard errors. neutral pictures, $M=5.16, S D=.31, F(1,23)=5.34, p<.05$, partial $\eta^{2}=.19, \mathrm{Cl}_{95}=.1,1.82$. As predicted, the $50 / 50$ condition, $M=6.13, S D=.27$, resulted in better recall than the 99/ 1 condition, $M=4.98, S D=.34, F(1,23)=10.98, p<.01$, partial $\eta^{2}=.32, \mathrm{Cl}_{95}=.50,2.16$. The interaction was not significant, $p>.10$. Crucially, there was no significant difference in memory between the two critical conditions, emotional pictures under 99/1 and neutral pictures under $50 / 50, t<1$, Cohen's $d=.18, \mathrm{Cl}_{95}=-1.55,0.80$. Note that recall of neutral pictures in these well-matched conditions was numerically higher than recall of emotional pictures, so the failure to obtain EEM in this comparison cannot be attributed to insufficient power.

\section{Discussion}

As predicted, regardless of the instructions participants received about how to divide their voluntary attention resources, emotional pictures captured more attention than neutral ones in both the 99/1 and the 50/50 conditions and EEM was manifested in both. EEM was abolished only when the amount of attention allocated to emotional and neutral pictures was equivalent, in the comparison of emotional 99/1 and neutral 50/50 picture sets. This comparison is the first to control organization, distinctiveness and attention simultaneously, and the first to show that EEM can be abolished under DA conditions. The finding that EEM was absent in the critical comparison is a null finding with obvious drawbacks for interpretation. However, the fact that neutral items were recalled slightly better than emotional items, and the magnitude of the effect of emotion on attention and memory within each instruction condition, speaks against a low power interpretation of these results. The theoretical implication of this pattern of results is that attention is necessary to account for immediate EEM.

Beyond its theoretical significance, this latter result also has practical implications. DA conditions resemble frequent real-life situations in which people perform two tasks at the same time. Indeed, emotional stimuli are often encountered in situations in which attending them conflicts with other goals, for instance, when trying to work despite the sound of a crying child. The current results suggest that although memory for emotional events is often better than memory for neutral events when people are engaged in many things at the same time, this may not always be the case when a series of events that are all emotionally arousing.

Previous work has shown that when emotional stimuli were attended as much as related-neutral stimuli but were higher in primary distinctiveness, they were recalled more frequently than neutral stimuli (Talmi, Schimmack, et al., 2007, Experiment 2). The present Experiment 2 employed the same paradigm as in that previous study but controlled primary distinctiveness, and managed to abolish EEM. Although comparisons across experiments should be interpreted with caution, this suggests that the EEM in our previous study likely stemmed from the higher primary distinctiveness of emotional stimuli, rather than from a direct effect of arousal. 


\section{General discussion}

What accounts for good immediate memory for emotional items? In real life, events that provoke an emotional response are typically related to each other, stand out relative to a background of less emotional items, and attract attention. The experiments reported here demonstrated that these three cognitive factors - organization, distinctiveness, and attention - contribute to EEM when memory is tested soon after study. When any of these is greater for emotional relative to neutral stimuli, EEM appears.

The present study confirms previous work, which had already demonstrated that organization and distinctiveness are necessary to account for EEM (Dewhurst \& Parry, 2000; Hadley \& Mackay, 2006; Talmi, Luk, et al., 2007), and adds a conclusive demonstration that attention is also necessary. Because organization and distinctiveness were controlled in the comparison of negative and related neutral pictures in the DA-blocked condition, but emotional stimuli were better attended, we could conclusively attribute the EEM obtained in this condition to the effect of attention. Indeed, attention mediated the effect of arousal on memory. When, in addition to organization and distinctiveness, attention was also controlled - in the full attention condition in Experiment 1 and the critical conditions in Experiment 2 - in Experiment 2, EEM disappeared.

With regard to the role or organization, in Experiment 1 the comparison of negative and random neutral pictures that were encoded under FA revealed EEM. Because primary distinctiveness and possibly attention were controlled in this comparison, the EEM could be attributed to the effect of organization. With regards to the role of distinctiveness, arousal ratings, which likely also indexed primary distinctiveness, predicted EEM under mixed-DA in Experiment 1. Furthermore, comparing the results of the matched attention conditions in Experiment 2 with those reported by Talmi, Schimmack, et al. (2007, Experiment 2) suggest that primary distinctiveness will produce EEM even when organization and attention are controlled.

Our results establish the cognitive account of EEM by integrating across a host of previous suggestions. They emphasize the importance of considering the interplay between different cognitive factors instead of examining any one of them in isolation, and show that the three factors we have considered provide a necessary and sufficient account for immediate EEM. These results explain why emotional memory is usually so good but also indicate that there may be real-life situations when a series of emotional events will be recalled no better than a series of neutral events.

Current results replicate and extend those of Schmidt and Saari (2007). Like us, these authors obtained EEM across both mixed and blocked-sets study conditions. Because the orienting task they used was a neutral task which was deemed primary, and suffered when stimuli were emotional, their encoding conditions resembled our own divided-attention conditions. This replication is encouraging because the emotional picture stimuli used here are arguably more arousing (De Houwer \& Hermans, 1994; Kensinger \& Schacter, 2006) than the taboo words employed by Schmidt and Saari. Current results extend those of Schmidt and Saari because here we demonstrated formally that when other factors are controlled, enhanced attention to emotional stimuli explains enhanced memory for them. This was evident in our mediator analysis results where attention mediated the effect of arousal on memory under DA-blocked, and in the critical comparison in Experiment 2, where EEM was obtained only when attention was not controlled but abolished when it was.

While current results show that the memory advantage of emotional over neutral stimuli depends on organization, distinctiveness, and attention, it is possible that memory for emotional stimuli utilizes different cognitive and neural mechanisms than those that underpin memory for neutral stimuli. This hypothesis was supported by an fMRI study which employed an encoding condition that resembled the FA-blocked condition in Experiment 1 (Sommer et al., 2008), and likewise found that memory for emotional and neutral items was equivalent. Nevertheless, activation in the amygdala and hippocampus correlated with immediate memory for emotional, but not neutral, stimuli. The interpretation of these intriguing results is complicated, however, because the activation of the amygdala and hippocampus could be due to the beginning of a differential consolidation process rather than to differential drivers of immediate EEM. Immediate memory data from patients with lesions in the amygdala, which could shed light on this causal link, is scant and mixed (Hurlemann et al., 2007; Labar \& Phelps, 1998; Phelps et al., 1998; Strange et al., 2003). Future research is needed to decide whether brain lesions dissociate immediate emotional and neutral memory.

Work in animal models shows that the modulation model cannot account for immediate long-term emotional memory. For instance, in the step-down one-trial inhibitory avoidance learning paradigm an animal is placed in an aversive location, such as a high, exposed platform, from which it can escape by stepping down. When the animal reaches the apparently safer location, however, it receives an electric shock. Memory is then tested by placing the animal on the platform again and measuring step-down latency. Noradrenaline infusion into the amygdala immediately after encoding mimics the effects of endogenous arousal on the modulation mechanism, and this treatment enhanced memory when it was tested $24 \mathrm{~h}$ after study. However, the treatment was no different to a control infusion of saline when memory was tested earlier, $1.5 \mathrm{~h}$ after study (Bianchin et al., 1999). In another study, a passive avoidance task measured an animal's hesitation to drink water following a previous experience of receiving an electric shock in the same location. Infusion of noradrenaline into the amygdala induced the animal to drink much sooner than infusion of saline when memory was tested $24 \mathrm{~h}$ after study, indicating enhanced memory for the shock, but there was no difference between the treatments when memory was tested $30 \mathrm{~min}$ after study (Ellis \& Kesner, 1983). Similarly, stimulation of the basolateral amygdala influences late-LTP but not earlyLTP in the dentate gyrus (Frey et al., 2001) and post-encoding behavioral inductions of arousal help sustain LTP in the dentate gyrus but do not influence LTP within the first hour 
after study (Seidenbecher et al., 1997). Indeed, because molecular pharmacology research in animal models often reveals dissociations between immediate and delayed memory (Izquierdo et al., 2002), it would not be surprising if immediate and delayed EEM stemmed from different mechanisms.

The cognitive account of immediate EEM could guide future work in animals to elucidate the neurophysiological mechanisms of immediate EEM, which is currently relatively unknown. For instance, what is the neural mechanism for the translation of enhanced attention allocation to emotional items to immediate EEM? One structure that may be important is the central nucleus of the amygdala, which is involved in enhanced attention allocation to salient events (Holland \& Gallagher, 1999). Recent animal work also reveals that noradrenaline projections from the locus coeruleus modulate hippocampal long-term depression and help select more salient information for encoding (Lemon, Aydin-Abidin, Funke, \& Manahan-Vaughan, 2009). Future research could explore whether these effects account for immediate EEM in animals, how they are related to the cognitive factors highlighted here, and how they interact with the well-known modulatory effects of the basolateral nucleus of the amygdala on long-term consolidation in the hippocampus (McGaugh, 2004).

Current findings could also be interpreted as presenting a challenge for the modulation model. Could attention, distinctiveness and organization - which fully accounted for immediate EEM in our study - also account for delayed EEM? This question is important because delayed tests of EEM have never, to our knowledge, controlled all three factors together. Although the rare, but theoretically significant, finding of delayed EEM in the absence of immediate EEM (Kleinsmith \& Kaplan, 1964; Sharot \& Phelps, 2004; Walker \& Tarte, 1963) is often interpreted as contradicting this hypothesis, the possibility remains that certain cognitive effects have a larger influence after a delay (Alba \& Hasher, 1983; Loftus, Schooler, \& Wagenaar, 1985). If true, this could perhaps explain the theoretically important findings that delayed EEM is correlated more strongly with the connectivity between the amygdala and hippocampus (Ritchey, Dolcos, \& Cabeza, 2008). This question, and the possible interaction of the cognitive factors with long-term memory consolidation, is therefore an important avenue for future research of emotion-enhanced memory.

\section{Acknowledgments}

This work was partly supported by NSERC Grant \# RGPIN 8347-08 Fund 454119 to M. Moscovitch. The author thanks M. Moscovitch for many fruitful discussions, P. Davidson for his assistance in stimulus development, S. Garnett and B. Renwick for help in data collection, L.A. Jones, M. Cohn and A.R. Mayes for comments on a draft of this manuscript.

\section{References}

Adolphs, R., Tranel, D., \& Buchanan, T. W. (2005). Amygdala damage impairs emotional memory for gist but not details of complex stimuli. Nature Neuroscience, 8, 512-518.
Alba, J. W., \& Hasher, L. (1983). Is memory schematic? Psychological Bulletin, 93, 203-231.

Algom, D., Chajut, E., \& Lev, S. (2004). A rational look at the emotional stroop phenomenon: A generic slowdown, not a stroop effect. Journal of Experimental Psychology - General, 133, 323-338.

Anderson, A. K. (2005). Affective influences on the attentional dynamics supporting awareness. Journal of Experimental Psychology - General, 134, 258-281.

Baron, R. M., \& Kenny, D. A. (1986). The moderator-mediator variable distinction in social psychological research: Conceptual, strategic, and statistical considerations. Journal of Personality and Social Psychology, 51, 1173-1182.

Bianchin, M., Mello e Souza, T., Medina, J. H., \& Izquierdo, I. (1999). The amygdala is involved in the modulation of long-term memory, but not in working or short-term memory. Neurobiology of Learning and Memory, 71, 127-131.

Bradley, M. M., Greenwald, M. K., Petry, M. C., \& Lang, P. J. (1992). Remembering pictures - Pleasure and arousal in memory. Journal of Experimental Psychology - Learning Memory and Cognition, 18, 379-390.

Bradley, M. M., \& Lang, P. J. (1994). Measuring emotion: The selfassessment manikin and the semantic differential. Journal of Behavior Therapy and Experimental Psychiatry, 25, 49-59.

Buchanan, T. W., Etzel, J. A., Adolphs, R., \& Tranel, D. (2006). The influence of autonomic arousal and semantic relatedness on memory for emotional words. International Journal of Psychophysiology, 61, 26-33.

Cahill, L., \& McGaugh, J. L. (1998). Mechanisms of emotional arousal and lasting declarative memory. Trends in Neurosciences, 21, 294-299.

Christianson, S. A., Loftus, E. F., Hoffman, H., \& Loftus, G. R. (1991). Eye fixations and memory for emotional events. Journal of Experimental Psychology - Learning Memory and Cognition, 17, 693-701.

Craik, F. I. M., Govoni, R., Naveh-Benjamin, M., \& Anderson, N. D. (1996). The effects of divided attention on encoding and retrieval processes in human memory. Journal of Experimental Psychology - General, 125, 159-180.

Craik, F. I. M., \& Kester, J. D. (1999). Divided attention and memory: Impairment of processing or consolidation? In Memory, consciousness, and the brain: The Tallinn conference (pp. 38-51). Philadelphia, PA: Psychology Press.

De Houwer, J., \& Hermans, D. (1994). Differences in the affective processing of words and pictures. Cognition E Emotion, 8, 1-20.

Dewhurst, S. A., \& Parry, L. A. (2000). Emotionality, distinctiveness and recollective experience. European Journal of Cognitive Psychology, 12, 541-551.

Dolan, R. J. (2002). Emotion, cognition, and behavior. Science, 298, 1191-1194.

Einstein, G. O., \& Hunt, R. R. (1980). Levels of processing and organization - Additive effects of individual-item and relational processing. Journal of Experimental Psychology - Human Learning and Memory, 6, 588-598.

Ellis, M. E., \& Kesner, R. P. (1983). The noradrenergic system of the amygdala and aversive information-processing. Behavioral Neuroscience, 97, 399-415.

Eysenck, M. W., \& Eysenck, M. C. (1979). Processing depth, elaboration of encoding, memory stores, and expended processing capacity. Journal of Experimental Psychology - Human Learning and Memory, 5, 472-484.

Faul, F., Erdfelder, E., Lang, A. G., \& Buchner, A. (2007). G*Power 3: A flexible statistical power analysis program for the social, behavioral, and biomedical sciences. Behavior Research Methods, 39, 175-191.

Frey, S., Bergado-Rosado, J., Seidenbecher, T., Pape, H. C., \& Frey, J. U. (2001). Reinforcement of early long-term potentiation (early-LTP) in dentate gyrus by stimulation of the basolateral amygdala: Heterosynaptic induction mechanisms of late-LTP. Journal of Neuroscience, 21, 3697-3703.

Hadley, C. B., \& Mackay, D. G. (2006). Does emotion help or hinder immediate memory? Arousal versus priority-binding mechanisms. Journal of Experimental Psychology - Learning Memory and Cognition, 32, 79-88.

Holland, P. C., \& Gallagher, M. (1999). Amygdala circuitry in attentional and representational processes. Trends in Cognitive Sciences, 3, 65-73.

Hunt, R. R., \& McDaniel, M. A. (1993). The enigma of organization and distinctiveness. Journal of Memory E Language, 32, 421-445.

Hunt, R. R., \& Seta, C. E. (1984). Category size effects in recall - The roles of relational and individual item information. Journal of Experimental Psychology - Learning Memory and Cognition, 10, 454-464.

Hunt, R. R., \& Worthen, J. B. (2006). Memory and distinctiveness. Oxford: Oxford University Press.

Hurlemann, R., Wagner, M., Hawellek, B., Reich, H., Pieperhoff, P., Amunts, K., et al. (2007). Amygdala control of emotion-induced forgetting and 
remembering: Evidence from Urbach-Wiethe disease Neuropsychologia, 45, 877-884.

Izquierdo, L. A., Barros, D. M., Vianna, M. R., Coitinho, A., deDavid, e Silva, Choi, H., et al. (2002). Molecular pharmacological dissection of shortand long-term memory. Cellular and Molecular Neurobiology, 22, 269-287.

Kahneman, D. (1973). Attention and effort. Englewood Cliffs, NJ: PrenticeHall.

Kensinger, E. A. (2004). Remembering emotional experiences: The contribution of valence and arousal. Reviews in the Neurosciences, 15 , 241-251.

Kensinger, E. A., \& Corkin, S. (2004). Two routes to emotional memory: Distinct neural processes for valence and arousal. Proceedings of the National Academy of Sciences of the United States of America, 101, 3310-3315.

Kensinger, E. A., \& Schacter, D. L. (2006). Processing emotional pictures and words: Effects of valence and arousal. Cognitive Affective Behavioral Neuroscience, 6, 110-126.

Kern, R. P., Libkuman, T. M., Otani, H., \& Holmes, K. (2005). Emotional stimuli, divided attention, and memory. Emotion, 5, 408-417.

Kerr, B. (1973). Processing demands during mental operations. Memory \& Cognition, 1, 401-412.

Kleinsmith, L. J., \& Kaplan, S. (1964). Interaction of arousal + recall interval in nonsense syllable paired-associate learning. Journal of Experimental Psychology, 67, 124-126.

Labar, K. S., \& Cabeza, R. (2006). Cognitive neuroscience of emotional memory. Nature Reviews Neuroscience, 7, 54-64.

Labar, K. S., \& Phelps, E. A. (1998). Arousal-mediated memory consolidation: Role of the medial temporal lobe in humans. Psychological Science, 9, 490-493.

Lang, P. J., Bradley, M. M., \& Cuthbert, B. N. (2005). International affective picture system (IAPS): Digitized photographs, instruction manual, and affective ratings (tech. rep. No. A-6). 2005. Gainesville, University of Florida, Center for Research in Psychophysiology.

Lemon, N., Aydin-Abidin, S., Funke, K., \& Manahan-Vaughan, D. (2009). Locus coeruleus activation facilitates memory encoding and induces hippocampal LTD that depends on beta-adrenergic receptor activation. Cerebral Cortex, 19, 2827-2837.

Loftus, E. F., Schooler, J. W., \& Wagenaar, W. A. (1985). The fate of memory - Comment. Journal of Experimental Psychology - General, 114, 375-380.

Mackay, D. G., Shafto, M., Taylor, J. K., Marian, D. E., Abrams, L., \& Dyer, J. R. (2004). Relations between emotion, memory, and attention: Evidence from taboo stroop, lexical decision, and immediate memory tasks. Memory \& Cognition, 32, 474-488.

MacKinnon, D. P., Lockwood, C. M., Hoffman, J. M., West, S. G., \& Sheets, V. (2002). A comparison of methods to test mediation and other intervening variable effects. Psychological Methods, 7, 83-104.

Majerus, S., \& D'Argembeau, A. (2011). Verbal short-term memory reflects the organization of long-term memory: Further evidence from shortterm memory for emotional words. Journal of Memory and Language, $64,181-197$.

Mandler, G. (1967). Organization and memory. Oxford, England: Academic Press.

Maratos, E. J., Allan, K., \& Rugg, M. D. (2000). Recognition memory for emotionally negative and neutral words: An ERP study. Neuropsychologia, 38, 1452-1465.

Mather, M. (2007). Emotional arousal and memory binding an objectbased framework. Perspectives on Psychological Science, 2, 33-52.

McDaniel, M. A., Dornburg, C. C., \& Guynn, M. J. (2005). Disentangling encoding versus retrieval explanations of the bizarreness effect: Implications for distinctiveness. Memory \& Cognition, 33, 270-279.

McDaniel, M. A., Einstein, G. O., Delosh, E. L., May, C. P., \& Brady, P. (1995). The bizarreness effect - Its not surprising, its complex. Journal of Experimental Psychology - Learning Memory and Cognition, 21 422-435.

McGaugh, J. L. (2004). The amygdala modulates the consolidation of memories of emotionally arousing experiences. Annual Review of Neuroscience, 27, 1-28.

Mitchell, D. B., \& Hunt, R. R. (1989). How much effort should be devoted to memory. Memory \& Cognition, 17, 337-348.

Mogg, K., Bradley, B. P., de Bono, J., \& Painter, M. (1997). Time course of attentional bias for threat information in non-clinical anxiety. Behavioral Research and Therapy, 35, 297-303.

Monnier, C., \& Syssau, A. (2008). Semantic contribution to verbal shortterm memory: Are pleasant words easier to remember than neutral words in serial recall and serial recognition? Memory \& Cognition, 36, $35-42$.

Murty, V. P., Ritchey, M., Adcock, R. A., \& Labar, K. S. (2010). fMRI studies of successful emotional memory encoding: A quantitative metaanalysis. Neuropsychologia, 48, 3459-3469.

Phelps, E. A., Labar, K. S., Anderson, A. K., O’Connor, K. J., Fulbright, R. K., \& Spencer, D. D. (1998). Specifying the contributions of the human amygdala to emotional memory: A case study. Neurocase, 4, 527-540.

Preacher, K. J., \& Hayes, A. F. (2004). SPSS and SAS procedures for estimating indirect effects in simple mediation models. Behavior Research Methods, Instruments, \& Computers, 36, 717-731.

Ritchey, M., Bessette-Symons, B., Hayes, S. M., \& Cabeza, R. (2011). Emotion processing in the aging brain is modulated by semantic elaboration. Neuropsychologia, 49, 640-650.

Ritchey, M., Dolcos, F., \& Cabeza, R. (2008). Role of amygdala connectivity in the persistence of emotional memories over time: An event-related fMRI investigation. Cerebral Cortex, 18, 2494-2504.

Schimmack, U. (2005). Attentional interference effects of emotional pictures: Threat, negativity, or arousal? Emotion, 5, 55-66.

Schmidt, S. R. (1991). Can we have a distinctive theory of memory? Memory \& Cognition, 19, 523-542.

Schmidt, S. R. (1994). Effects of humor on sentence memory. Journal of Experimental Psychology - Learning Memory and Cognition, 20, 953-967.

Schmidt, S. R. (2002). Outstanding memories: The positive and negative effects of nudes on memory. Journal of Experimental Psychology Learning Memory and Cognition, 28, 353-361.

Schmidt, S. R., \& Saari, B. (2007). The emotional memory effect: Differential processing or item distinctiveness? Memory \& Cognition, 35, 1905-1916

Seidenbecher, T., Reymann, K. G., \& Balschun, D. (1997). A post-tetanic time window for the reinforcement of long-term potentiation by appetitive and aversive stimuli. Proceedings of the National Academy of Sciences of the United States of America, 94, 1494-1499.

Sharot, T., \& Phelps, E. A. (2004). How arousal modulates memory: Disentangling the effects of attention and retention. Cognitive Affective Behavioral Neuroscience, 4, 294-306.

Smith, J. C., Bradley, M. M., \& Lang, P. J. (2005). State anxiety and affective physiology: Effects of sustained exposure to affective pictures. Biological Psychology, 69, 247-260.

Sommer, T., Glascher, J., Moritz, S., \& Buchel, C. (2008). Emotional enhancement effect of memory: Removing the influence of cognitive factors. Learning \& Memory, 15, 569-573.

Strange, B. A., Hurlemann, R., \& Dolan, R. J. (2003). An emotion-induced retrograde amnesia in humans is amygdala- and beta-adrenergic dependent. Proceedings of the National Academy of Sciences of the United States of America, 100, 13626-13631.

Talmi, D., Grady, C. L., Goshen-Gottstein, Y., \& Moscovitch, M. (2005). Neuroimaging the serial position curve. A test of single-store versus dual-store models. Psychological Science, 16, 716-723.

Talmi, D., Luk, B. T. C., McGarry, L. M., \& Moscovitch, M. (2007). The contribution of relatedness and distinctiveness to emotionallyenhanced memory. Journal of Memory and Language, 56, 555-574.

Talmi, D., \& Moscovitch, M. (2004). Can semantic relatedness explain the enhancement of memory for emotional words? Memory \& Cognition, $32,742-751$

Talmi, D., Schimmack, U., Paterson, T., \& Moscovitch, M. (2007). The role of attention and relatedness in emotionally enhanced memory. Emotion, 7, 89-102.

Tomlinson, T. D., Huber, D. E., Rieth, C. A., \& Davelaar, E. J. (2009). An interference account of cue-independent forgetting in the no-think paradigm. Proceedings of the National Academy of Sciences of the United States of America, 106, 15588-15593.

Tulving, E., \& Pearlstone, Z. (1966). Availability versus accessibility of information in memory for words. Journal of Verbal Learning and Verbal Behavior, 5, 381-391.

Vuilleumier, P. (2005). How brains beware: Neural mechanisms of emotional attention. Trends in Cognitive Sciences, 9, 585-594.

Walker, E. L., \& Tarte, R. D. (1963). Memory storage as a function of arousal and time with homogeneous and heterogeneous lists. Journal of Verbal Learning and Verbal Behavior, 2, 113-119.

Wessel, I., van der, K. P., \& Merckelbach, H. (2000). Differential recall of central and peripheral details of emotional slides is not a stable phenomenon. Memory, 8, 95-109.

Windmann, S. \& Kutas, M. (2001). Electrophysiological correlates of emotion-induced recognition bias. Journal of Cognitive Neuroscience, $13,577-592$ 